\title{
Links between Lung Dysfunction and Glucose Metabolism Dysregulation: Does Lung Dysfunction Represent a Systemic Disorder?
}

\author{
Hiroo Wada ${ }^{1}$, Shin-ichi Hagiwara ${ }^{2}$, Yorihiro Yamamoto $^{3}$ and Hajime Takizawa ${ }^{1}$ \\ Key words: vital capacity (VC), forced expiratory volume in one second (FEV1), COPD, fatty acid, diabetes
}

(Intern Med 53: 2413-2414, 2014)

(DOI: 10.2169/internalmedicine.53.2700)

Chronic obstructive pulmonary disease (COPD) is regarded as a systemic inflammatory disorder with extrapulmonary manifestations, such as malnutrition, osteoporosis, depression, and vascular events. Compromised lung function may be an indicator of systemic conditions in patients with COPD. However, it remains to be clarified whether or not lung function can be used to assess systemic disease in healthy individuals. This question has been addressed by several cross-sectional epidemiologic studies, which showed that lung dysfunction is associated with glucose metabolism dysregulation (1-6). These findings raised several questions regarding the association between lung function and diabetes: namely, is lung dysfunction exclusively an event in the lung, or is it systemically associated with disorders of other organs? Does deteriorated lung function result from diabetes or influence its development? What are the key molecules? What is the clinical and scientific significance of this link?

A retrospective and longitudinal observation by Oda, published in this issue of Internal Medicine, provides evidence for the link between lung dysfunction and diabetes. This study included 1,704 men and 1,016 women in Japan and determined factors that were significantly associated with diabetes development. The results of a multivariate analysis revealed that high-sensitivity $\mathrm{C}$-reactive protein (hs-CRP) and percent vital capacity (\%VC) were independently associated with the development of incident diabetes in women within four years (7). Although the lowest tertile of \% VC in this cohort failed to predict the groups at higher risk of developing diabetes, this study was the first attempt at elucidating the association between lung dysfunction and impaired glucose metabolism in East Asian women. These results were supported by those of other longitudinal studies, further demonstrating that lung dysfunction precedes glucose metabolism dysregulation (8-10). However, these studies, did not reach the conclusion that lung dysfunction led to glucose metabolism dysregulation, because participants who were already diabetic were excluded from the analyses (7-10). In fact, several studies have shown that diabetic individuals are more likely to develop lung dysfunction $(2,3,5)$. In addition, based on other analyses of the same cohort, Oda et al. previously reported that lower VC was associated with diabetes in lean Japanese men (6), suggesting that the association is present in both sexes. Therefore, the relationship between lung dysfunction and glucose metabolism dysregulation might not be directly related in terms of cause and effect. Rather, these controversial results indicate that both lung dysfunction and glucose metabolism dysregulation share some common pathogenetic pathways or environmental factors. Environmental air pollution, for example, seems to be associated with both deteriorated lung function (11-13) and abnormal glucose metabolism $(14,15)$. Exposure to air pollutants, including diesel exhaust particle (DEP), exacerbates inflammatory reactions in cells and induces airway inflammation in mouse models and asthmatic patients, partly via the suppression of nuclear factor-like 2 (Nrf2)-linked signals (13). On the other hand, uncoupling protein 1 (UCP-1) expression is suppressed (14) and hepatic glucose metabolism is impaired (15) by exposure to air pollution, particulate matter (PM) 2.5, and either of these events results in glucose intolerance.

One of the candidate molecular events that occur in response to certain environmental changes and accounts for lung dysfunction and glucose metabolism dysregulation, is the up-regulation of SIRT-1, the mammalian ortholog of sirtuin (16-18). Reduced SIRT-1 expression and/or activity is

${ }^{1}$ Kyorin University School of Medicine, Japan, ${ }^{2}$ Health Care Centre, Honda Engineering Co., Ltd., Japan and ${ }^{3}$ Faculty of Biotechnology, Tokyo University of Technology, Japan

Received for publication February 14, 2014; Accepted for publication April 2, 2014

Correspondence to Dr. Hiroo Wada, wadagh@ks.kyorin-u.ac.jp 
caused by cigarette smoke exposure and is associated with accelerated ageing of the lung $(16,17)$, Conversely, its enhancement, which occurs in response to calorie restriction, is associated with increased longevity (18). Animal model studies demonstrated that mice exposed to cigarette smoke exhibited reduced SIRT-1 levels, together with enhanced neutrophilic inflammation and increased matrix metalloproteinase (MMP)-9 levels (17). This neutrophilic inflammation was attenuated in mice treated with a SIRT-1 activator. Similarly, patients with COPD showed reduced SIRT-1 expression (18). Animal studies $(19,20)$ and human clinical trials $(21,22)$ have both demonstrated that treatment with SIRT-1 activator has a beneficial effect on glucose metabolism regulation.

Reflecting these molecular events, it is well known that several proinflammatory cytokines and mediators, including tumor necrosis factor (TNF)-alpha, interleukin (IL)-1, IL-6, leptin, adiponectin, and resistin, are in common between COPD and diabetes (23), which will provide some insight into their pre-disease stages. In addition to these proinflammatory molecules, MMPs were reported to be increased both in COPD and diabetic patients; these proteases digest extracellular matrix, which is also a characteristic of the ageing process (16-18).

Collectively, these epidemiologic observations and molecular data led to the hypothesis that lung dysfunction reflects systemic disorders. To test this, health check up data and biomarker results for molecular events in a body should have been collected and analyzed. However, Oda's study still has a very important and universal message that lung function may reflect not only the direct environmental effects on the lung, but also that of systemic processes in the body. The close link between lung dysfunction and glucose metabolism dysregulation will increase the understanding of both conditions, and further studies on this link are likely to identify novel molecular mechanisms of the development of lung disease and glucose metabolism abnormalities. Ultimately, such research will clarify what lifestyles or behaviors will favorably affect our health and longevity and which biomarkers should be used to estimate them.

Author's disclosure of potential Conflicts of Interest (COI). Shin-ichi Hagiwara: Employment, Honda Engineering.

\section{References}

1. Walter RE, Beiser A, Givelber RJ, O'Connor GT, Gottlieb DJ. Association between glycemic state and lung function: Framingham Heart Study. Am J Respir Crit Care Med 2003 167: 911-916, 2003.

2. Yeh HC, Punjabi NM, Wang NY, et al. Cross-sectional and prospective study of lung function in adults with type 2 diabetes: the Atherosclerosis Risk in Communities (ARIC) Study. Diabetes Care 31: 741-746, 2008.

3. Lange P, Parner J, Schnohr P, Jensen G. Copenhagen City Heart
Study: longitudinal analysis of ventilator capacity in diabetic and non-diabetic adults. Eur Respir J 20: 1406-1412, 2002.

4. Laolor DA, Ebrahim S, Smith GD. Associations of measures of lung function with insulin resistance and type 2 diabetes: findings form the British Women's Heart and Health Study. Diabetologia 47: 195-203, 2004.

5. Litonjua AA, Lazarus R, Sparrow D, Demolles D, Weiss ST. Lung function in type 2 diabetes: the Normative Aging Study. Respir Med 99: 1583-1590, 2005.

6. Oda E, Kawai R. Low vital capacity is associated with diabetes depite inverse relationship with metabolic risk factors in lean Japanese men. Intern Med 48: 1201-1207, 2009.

7. Oda E. Low vital capacity was found to be associated with incident diabetes in health screening population of japanese women. Intern Med 53: 2463-2469, 2014.

8. Ford ES, Mannino D. National Health and Nutrition Examination Survey Epidemiologic Follow-up Study. Prospective association between lung function and the incidence of diabetes: findings from the National Heatlh and Nutrition Examiniation Survey Epidemiologic Followup Sutdy. Diabetes Care 27: 2966-2970, 2004.

9. Engstrom G, Hedbad B, Nisson P, Wollmer P, Berglund G, Janzon L. Lung function, insulin resitance and incidence of cardiovascular diasese: a longitudinal cohort study. J Intern Med 2003 253: 574581, 2003.

10. Yeh HC, Punjabi NM, Wang NY, Pankow JS, Duncan BB, Brancati FL. Vital capacity as a predictor of incident type 2 diabetes: the atherosclerosis risk in communities study. Diabetes Care 28: 1472-1479, 2005.

11. Takizawa $\mathrm{H}$, Abe $\mathrm{S}$, Ohtoshi $\mathrm{T}$, et al. Disel exhaust particles upregulate expression of intercellular adhesion moeluce-1 (ICAM-1) in human bronchial epithelial cells. Clin Exp Immunol 120: 356362, 2000.

12. Li YJ, Shimizu T, Hirata Y, et al. EM, EM-703 inhibit NF- $\kappa B$ activation induced by oxidative stress from diesel exhaust particle in human bronchial epithelial cells: importance in IL-8 transcription. Pulm Pharmacol Ther 26: 318-324, 2003.

13. Li YJ, Takizawa H, Azuma A, et al. Nrf2 is closely related to allergic airway inflammatory responses induced by low-dose diesel exhaust particles in mice. Clin Immunol 137: 234-241, 2010.

14. Liu C, Ying Z, Harkema J, Sun Q, Rajagopalan S. Epidemiological and experimental linkes between airpllutaion and type 2 diabetes. Toxicol Pathol 41: 361-373, 2013.

15. Zheng Z, Xu X, Zhang X, et al. Exposure to ambient particulate matter induces a NASH-like phenotype and impairs hepatic glucose metabolism in an animal model. J Hepatol 58: 148-154, 2013.

16. Ito $\mathrm{K}$, Barnes PJ. COPD as a disease of accelerated lung aging. Chest 135: 173-180, 2009.

17. Nakamaru $\mathrm{Y}$, Vuppusetty $\mathrm{C}$, Wada $\mathrm{H}$, et al. A protein deacetylase SIRT1 is a negative regulator of metalloproteinase-9. FASEB J 23: 2810-2819, 2009.

18. Hubbard B, Sinclair DA. Small molecule SIRT1 activators for the treatment of aging and age-related diseases. Trend Pharmacol Sci 35: 146-154, 2014.

19. Yoshino J, Mills KF, Yoon MJ, Imai S. Nicotinamide mononucleotide, a key $\mathrm{NAD}^{+}$intermediate, treats the pathophysiology of dietand age-induced diabetes in mice. Cell Metab 14: 528-536, 2011.

20. Banks AS, Kon N, Knight C, et al. SirT1 gain of function increases energy efficiency and prevents diabetes in mice. Cell Metab 8: 333-341, 2008.

21. Vlassara H, Uribarri J, Cai W, et al. Effects of sevelamer on $\mathrm{HbA1c}$, inflammation, and advanced clycation end products in diabetic kidney disease. Clin J Am Soc Nephrol 7: 934-942, 2012.

22. Kitada M, Koya D. SIRT1 in type 2 diabetes: mechanisms and therapeutic potential. Diabetes Metab J 37: 315-325, 2013.

23. Mirrakhimov AE. Chronic obstructive pulmonary disease and glucose metabolism: a bitter sweet symphony. Cardiovasc Diabetol 11: 132, 2012.

(C) 2014 The Japanese Society of Internal Medicine http://www.naika.or.jp/imonline/index.html 